\title{
Efficient power allocation method for non orthogonal multiple access 5G systems
}

\author{
Maan A. S. Al-Adwany \\ Department of Computer and Information Engineering, Ninevah University, Iraq
}

\begin{tabular}{|c|c|}
\hline Article Info & ABSTRACT \\
\hline Article history: & \multirow{6}{*}{$\begin{array}{l}\text { One of the hot research topics for the upcoming } 5 \mathrm{G} \text { (fifth-generation) wireless } \\
\text { communication networks is the non orthogonal multiple access (NOMA) systems } \\
\text { where it have attracted both industrial and academic fields to improve the existing } \\
\text { spectral efficiency. In fact, the multiuser detection process for NOMA systems is } \\
\text { largely affected by the power distribution of the received signals. In this paper, a new } \\
\text { method has been proposed to control the transmit power among active users in one of } \\
\text { the promising NOMA systems; the interleave division multiple access (IDMA) which } \\
\text { has been adopted here for consideration. Unlike conventional methods, where tedious } \\
\text { mathematical computations are required; a simple and direct method has been derived } \\
\text { The proposed method has been applied to IDMA system with different FEC codes } \\
\text { The obtained results show that the proposed method outperforms the conventional one } \\
\text { as compared to optimal results. }\end{array}$} \\
\hline $\begin{array}{l}\text { Received Apr 10, } 2019 \\
\text { Revised Oct 19, } 2019 \\
\text { Accepted Oct 27, } 2019\end{array}$ & \\
\hline Keywords: & \\
\hline IDMA & \\
\hline NOMA & \\
\hline Power allocation & \\
\hline
\end{tabular}

Copyright (c) 2020 Insitute of Advanced Engineeering and Science. All rights reserved.

Corresponding Author:

Maan Ahmed Shehathah Al-Adwany,

Assistant Professor at Department of Computer and Information Engineering,

College of Electronics Engineering, Ninevah University, Mosul, Iraq.

Email: maanaladwany@yahoo.com

\section{INTRODUCTION}

Researchers have considered multiuser detection techniques for spread spectrum wireless communications systems and, recently, for non orthogonal multiple access (NOMA) systems. In fact, the multiuser detection process is largely affected by the power of the received signals [1-3]. Thus, managing these powers is crucial for interference suppression and the stability of the NOMA systems [4, 5]. In addition, one of the major design concerns is the power consumption reduction for mobile devices. Thus, minimization of total power received by uplink receiver is required while maintaining the SINR (signal to interference plus noise ratio) on certain desired level at final iteration of multiuser detector. That is so called the 'power allocation problem'. Recently, researchers spare no effort to solve the power allocation problem in NOMA system [1,2,6-9], while early researches on power allocation problem were equally important that can be found elsewhere [10-15].

Although the methods that have been proposed in the literatureare have involved difficult computations; they did not lead to exact solutions as one may expect. This, in turn, encouraged me to derive a simple method to solve power allocation problem in IDMA as one of the promising NOMA systems for $5 \mathrm{G}$. The contribution of this paper can be summerized with the following points:

(a) This paper presents a method which could be an efficient alternative to the existing power allocation methods for IDMA system.

(b) The proposed method draws its strength from being simple and direct. It depends only on the forward error correcting (FEC) rate to solve power allocation problem in IDMA system; whereas other methods usually use linear programing or complex analytical approaches. 
This paper is organized as follows: after the introduction, the IDMA uplink system is briefly described in section 2 in order for a reader to focus only on the power allocation problem which is presented in section 3 . Whereas a modelling of the proposed method is considered in section 4. Numerical results are then discussed in section 5. Finally, the conclusions are drawn in section 6.

\section{IDMA UPLINK SYSTEM}

Interleave Division Multiple Access (IDMA) is a promising non orthogonal multiple access system [16-19], so it has been adopted for consideration in this research. It can be considered as a special case of spread spectrum systems. In IDMA system the users are identified by their unique interleavers [20-27], while in spread spectrum systems such as CDMA the users are identified by their unique codes. The mechanism of the IDMA uplink system can be clarified according to the schematic diagram shown in Figure 1.

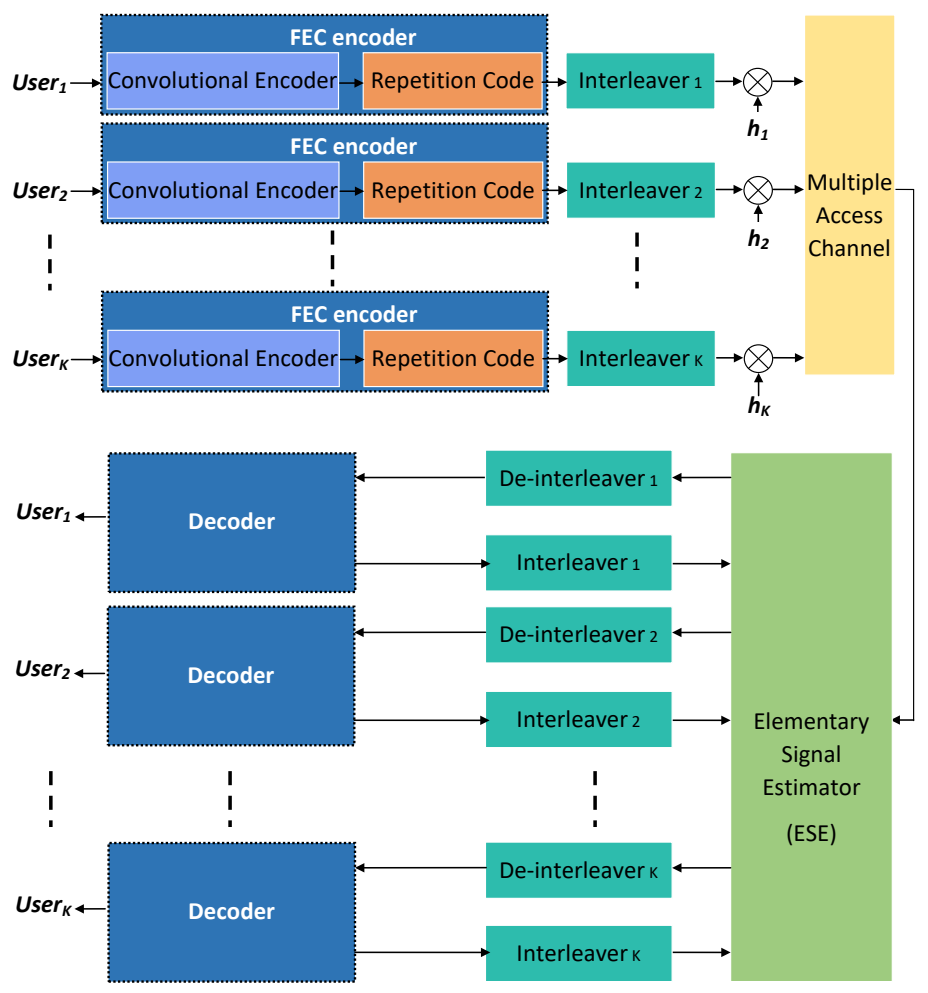

Figure 1. The structure of the IDMA system

At the transmit side, the information bits of each user are encoded using certain FEC (forward-errorcorrection) codes that are assumed the same for all users. Each FEC code is achieved by serial concatenation of convolutional code of rate $R_{c}$ along with a repetition code of rate $R_{p}$ resulting a total FEC code rate of $R_{t}=R_{c} R_{p}$. Consequently, the system can be considered to have a total spreading factor $S F=1 / R_{t}$. Figure 2. illustrates some well-known FEC codes that have been used in this paper. After that, the encoded bits (also called chips) are organized into frames each has length of 1024; to be prepared for the interleaving process where each user is assigned a unique interleaver. In IDMA, the key principle is that the user specific interleavers should have minimum cross correlation and must be generated randomly and independently. In fact, these user specific interleavers make a dispersion in the encoded sequences which produces an approximately uncorrelated adjacent chips; this in turn facilitates the receiver detection process. The interleavers at receiver and transmitter sides should be stored somewhere at the base station by utilizing a memory prepared for this purpose. Throughout the duration of the initial link setting-up stage, the base station and the mobile stations must exchange messages to tell each other about the employed interleavers. In this paper, in order to focus on the power allocation problem, it is assumed that the employed interleavers are optimum. 


\begin{tabular}{|c|c|c|c|c|c|c|c|c|}
\hline & \multicolumn{4}{|c|}{ Convolutional Code } & \multirow{2}{*}{$\begin{array}{c}\text { Repetition } \\
\text { Code Rate } \\
\quad\left(R_{p}\right)\end{array}$} & \multirow{2}{*}{$\begin{array}{c}\text { Total Code } \\
\text { Rate } \\
\left(R_{t}=R_{c} R_{p}\right)\end{array}$} & \multirow{2}{*}{$\begin{array}{c}\text { Total } \\
\text { Spreading } \\
\text { Factor } \\
S F=1 / R_{t}\end{array}$} & \multirow{2}{*}{$k_{\text {max }}$} \\
\hline & $\begin{array}{c}\text { Code } \\
\text { generator } \\
\text { (octal) }\end{array}$ & $\begin{array}{c}\text { Constraint } \\
\text { length }\end{array}$ & Feedback & $\begin{array}{c}\text { Code Rate } \\
\left(R_{c}\right)\end{array}$ & & & & \\
\hline FEC1 & $(237345)_{8}$ & 8 & $(237)_{8}$ & $1 / 2$ & $1 / 8$ & $1 / 16$ & 16 & 12 \\
\hline FEC2 & $(2731)_{8}$ & 5 & $(27)_{8}$ & $1 / 2$ & $1 / 8$ & $1 / 16$ & 16 & 13 \\
\hline $\mathrm{FEC3}$ & $\left(\begin{array}{ll}5 & 3\end{array}\right)_{8}$ & 3 & $(5)_{8}$ & $1 / 2$ & $1 / 8$ & $1 / 16$ & 16 & 18 \\
\hline FEC4 & $(2335)_{8}$ & 5 & $(23)_{8}$ & $1 / 2$ & $1 / 4$ & $1 / 8$ & 8 & 7 \\
\hline
\end{tabular}

Figure 2. Some FEC codes that were used in this research

The interleaver output is then scaled by coefficient for each user $h_{k}$; which is assumed to constitute the combined effect of the channel loss and power control factor. In other words, the distribution of $h_{k}^{2}$ can be fitted via power control process. The signals of all active users are assumed to be superimposed into a wireless flat fading channel. At the receive side, the receiver is composed of elementary signal estimator (ESE) along with a bank of $K$ decoders (DECs) corresponding to $K$ users. The DECs are a posteriori probability (APP) decoders. A related point to consider, is that the operation of the receiver is mainly based on multiple access channel and the FEC code constraints. The outcomes of the ESE and DECs are combined, via an iterative process, to retrieve the transmitted bits. It is suitable here to mention that we have designed and achieved the IDMA uplink system simulator using MATLAB, but it has not been included in this paper to avoid redundancy.

\section{PROBLEM FORMULATION}

As mentioned before, the performance of IDMA system can be enhanced through suitable power allocation. The proper power distribution among users will highly increase the chance for correct signal detection at the receiver. Strong signals are firstly detected. Consequently, their interference effect on weak signals can be correctly removed which in turn increase the chance of weak signal detection.

The main research question here is: how to allocate the power levels among users?. In general, the power allocation problem can be solved by dividing the total number of multiple access users into groups, where for each a certain power level is assigned. As a consequence, the former research question triggers the following three sub-questions:

(a) What is the proper size for each group?

(b) What is the proper power level for each group to achieve feasible target BER?

(c) If 1 and 2 are satisfied, has the total transmitted power $\left(P_{t}\right)$ from all active users been minimized as a result?

Early researches tried to solve this issue; for instance Schlegel et al. [28] proved that the minimum total power can be achieved when the groups have an equal number of users; so one can adopt this assumption as a foundation to meet other sub-questions. Therefore, the objective of this research becomes how to find the proper power level for each group so that the minimum total power condition is satisfied with feasible BER (typically $10^{-6}$ ).

\section{MODELLING OF PROPOSED METHOD}

Unlike the existing researches, this paper have revealed a new feature for FEC codes which can be utilized to solve the power allocation problem. The feature tells us that all FEC codes can support limitted number of users (users with same power level). However, exceeding this limit leads to degradation in system performance. Figure 3 shows the simulation results of the BER with number of equal power users for different FEC codes. One can observe that for each FEC code, a target BER of $10^{-6}$ can be achieved as long as the number of users do not exceed certain threshold say $k_{\max }$. Above this threshold, the BER jumps to infeasible values.

In this work, such a feature has been exploited to solve the power allocation problem. It is obvious, from the former figure, that $k_{\max }$ for the codes FEC1, FEC2, and FEC3, are 12, 13, and 18, respectively. 
Figure 2 demonstrates some FEC codes with their corresponding $k_{\max } \mathrm{s}$. To clarify our proposed method, it is suitable to discuss the following example. Assume an IDMA (NOMA) system with BPSK mapping of $S N R=10 d B$ and with the following specifications: the total number of users $(K)$ is 32 ; a FEC code consisting of convolutional code of rate $1 / 2$ serially concatenated with a repetition code of length 8 (i.e $R_{p}=1 / 8$ ). Thus the total FEC rate is $R_{t}=1 / 16$.

In order to distribute the power among active users; first, one should divide the total number of users $K$ into groups. We start with the case of 32 groups, each group consists of one user. Now, assume that the power set $\left\{p_{1}^{*} p_{2}^{*} \ldots p_{32}^{*}\right\}$ represents a solution to the power allocation problem. The power set should satisfy two conditions: a minimum total power; and convergence to feasible BER. By assuming that the uplink users have the same transmission rate, one can prove that the power set should follow a constant ratio according to [29]; hence:

$$
p_{2}^{*} / p_{1}^{*}=p_{3}^{*} / p_{2}^{*} \ldots=p_{K}^{*} / p_{K-1}^{*}=\Psi
$$

where $\Psi$ represents power ratio. Assume $p_{1}^{*}$ represents the minimum transmit power allowed for each user $\left(p_{\min }\right)$, hence the power set can be rewritten as:

$$
\left\{p_{\min } \beta p_{\min } \beta^{2} p_{\min } \ldots . . \beta^{31} p_{\min }\right\}
$$

or

$$
p_{\min }\left\{1 \beta \beta^{2} \ldots . \beta^{31}\right\}
$$

The power ratio $(\Psi)$ in this case is equal to $\beta$. It is worth mentioning that $p_{\min }$ can be determined according to the wireless system requirements. Thus, solution of the power allocation problem is mainly achieved by finding $\beta$. Since total power should be minimized, hence the power allocation problem can be solved by finding the minimum $\beta$ (i.e. $\beta_{\min }$ ), or for the sake of generality $\Psi_{\min }$.

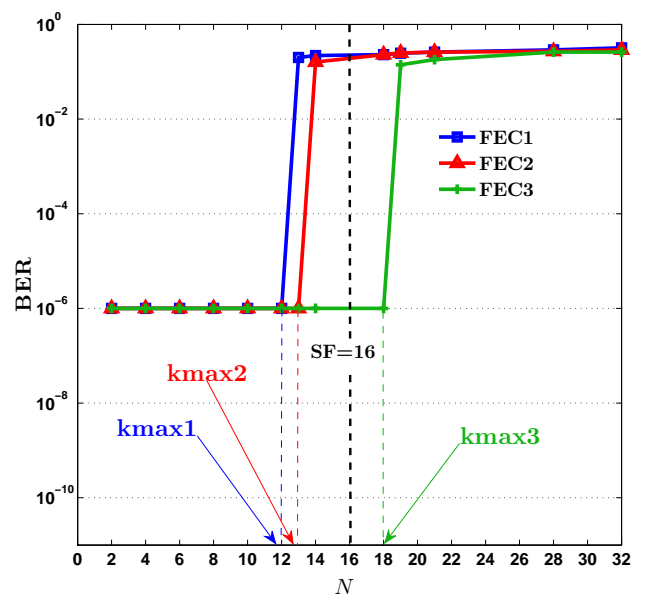

Figure 3. BER vs. number of equal power users

\subsection{Finding minimum power ratio $\left(\beta_{\min }\right)$}

Going forward to find $\beta_{\text {min }}$, assume that $g$ represents the number of users per group (or the group size). Thus, for the same total number of users $K=32$, in order to increase the group size from 1 to 2 one should raise the power ratio $\Psi$ from $\beta$ to $\beta^{2}$ as shown in Figure 4; starting from rightmost column . This is logically acceptable if one thinks of it as to ensure that each group will have enough power for converging to the target BER. By the same way, in order to increase $g$ to 4 , the power ratio $\Psi$ should be increased to $\beta^{4}$, and so on. Figure 4 shows the possible group sizes along with the corresponding power ratios. In general, one can observe that for certain group size $(g)$; the corresponding power ratio should be $\beta^{g}$. Figure 5 illustrates the effect of group size on the power ratio. It is obvious that as the group size increases, the required power ratio also increases. While for certain FEC code, as the group size approaches $k_{\text {max }}$, the required power ratio fairly increases. Thus, in order to avoid unwanted increase in total power, we recommend that the maximum group size should not approach $k_{\max }$ for given FEC code. 


\begin{tabular}{|c|c|c|c|c|}
\hline \multicolumn{5}{|c|}{ Total number of users $=32$} \\
\hline 2 groups & 4 groups & 8 groups & 16 groups & 32 groups \\
\hline$g=16$ users/group & $g=8$ users/group & $g=4$ users/group & $g=2$ users/group & $\mathrm{g}=1$ users/group \\
\hline Power Ratio $(\Psi)=\beta^{16}$ & Power Ratio $(\Psi)=\beta^{8}$ & Power Ratio $(\Psi)=\beta^{4}$ & Power Ratio $(\Psi)=\beta^{2}$ & Power Ratio $(\Psi)=\beta$ \\
\hline \multirow{16}{*}{ 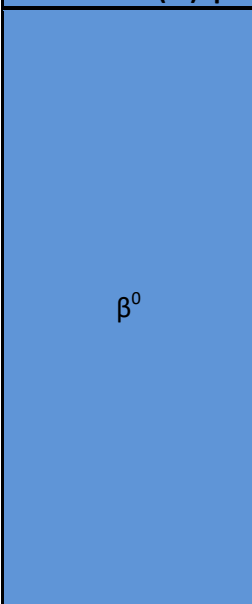 } & \multirow{8}{*}{ 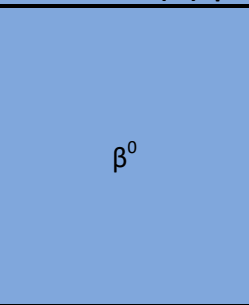 } & \multirow{4}{*}{$\beta^{0}$} & \multirow{2}{*}{$\beta^{0}$} & $\beta^{0}$ \\
\hline & & & & $\beta^{1}$ \\
\hline & & & \multirow{2}{*}{$\beta^{2}$} & $\beta^{2}$ \\
\hline & & & & $\beta^{3}$ \\
\hline & & \multirow{4}{*}{$\beta^{4}$} & \multirow{2}{*}{$\beta^{4}$} & $\beta^{4}$ \\
\hline & & & & $\beta^{5}$ \\
\hline & & & \multirow{2}{*}{$\beta^{6}$} & $\beta^{6}$ \\
\hline & & & & $\beta^{7}$ \\
\hline & \multirow{8}{*}{$\beta^{8}$} & \multirow{4}{*}{$\beta^{8}$} & \multirow{2}{*}{$\beta^{8}$} & $\beta^{8}$ \\
\hline & & & & $\beta^{9}$ \\
\hline & & & \multirow{2}{*}{$\beta^{10}$} & $\beta^{10}$ \\
\hline & & & & $\beta^{11}$ \\
\hline & & \multirow{4}{*}{$\beta^{12}$} & \multirow{2}{*}{$\beta^{12}$} & $\beta^{12}$ \\
\hline & & & & $\beta^{13}$ \\
\hline & & & \multirow{2}{*}{$\beta^{14}$} & $\beta^{14}$ \\
\hline & & & & $\beta^{15}$ \\
\hline \multirow{16}{*}{$\beta^{16}$} & \multirow{8}{*}{$\beta^{16}$} & \multirow{4}{*}{$\beta^{16}$} & \multirow{2}{*}{$\beta^{16}$} & $\beta^{16}$ \\
\hline & & & & $\beta^{17}$ \\
\hline & & & \multirow{2}{*}{$\beta^{18}$} & $\beta^{18}$ \\
\hline & & & & $\beta^{19}$ \\
\hline & & \multirow{4}{*}{$\beta^{20}$} & \multirow{2}{*}{$\beta^{20}$} & $\beta^{20}$ \\
\hline & & & & $\beta^{21}$ \\
\hline & & & \multirow{2}{*}{$\beta^{22}$} & $\beta^{22}$ \\
\hline & & & & $\beta^{23}$ \\
\hline & \multirow{8}{*}{$\beta^{24}$} & \multirow{4}{*}{$\beta^{24}$} & \multirow{2}{*}{$\beta^{24}$} & $\beta^{24}$ \\
\hline & & & & $\beta^{25}$ \\
\hline & & & \multirow{2}{*}{$\beta^{26}$} & $\beta^{26}$ \\
\hline & & & & $\beta^{27}$ \\
\hline & & & $\beta^{28}$ & $\beta^{28}$ \\
\hline & & $\Omega^{28}$ & & $\beta^{29}$ \\
\hline & & $P$ & $\beta^{30}$ & $\beta^{30}$ \\
\hline & & & $P$ & $\beta^{31}$ \\
\hline$P_{t 5}=g P_{\min }\left(\beta^{0}+\beta^{16}\right)$ & $\begin{array}{c}P_{t 4}=g P_{\min }\left(\beta^{0}+\right. \\
\left.\beta^{8}+\beta^{16}+\beta^{24}\right)\end{array}$ & $\begin{array}{l}P_{t 3}=g P_{\min }\left(\beta^{0}+\right. \\
\left.\beta^{4}+\beta^{8}+\ldots+\beta^{28}\right)\end{array}$ & $\begin{array}{l}P_{t 2}=g P_{\min }\left(\beta^{0}+\right. \\
\left.\beta^{2}+\beta^{4}+\ldots+\beta^{30}\right)\end{array}$ & $\begin{array}{l}P_{t 1}=g P_{\min }\left(\beta^{0}+\right. \\
\left.\beta^{1}+\beta^{2}+\ldots+\beta^{31}\right)\end{array}$ \\
\hline
\end{tabular}

Figure 4. Estimated power ratios for different group sizes

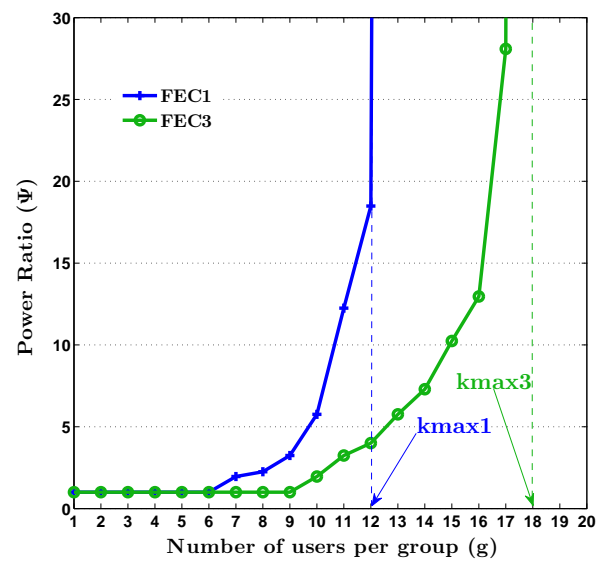

Figure 5. Power ratio vs. group size for different FEC codes 
The important note here is that for certain FEC code (say FEC3), the sum of the users' normalized powers should not exceed $k_{\max }$ as seen by the receiver; otherwise the BER jumps to infeasible values as shown in Figure 3. So;

$$
\frac{P_{\text {Total }}}{P_{\max }} \leq k_{\max }
$$

Now, assume that the number of groups is decreased from 32 down to 2. Thus, each of the resulting two groups would have the maximum number of users $g_{\max }$ (where $g_{\max }=16$ in our example). Hence, one can derive;

$$
\frac{\left(g_{\max } P_{\min }+g_{\max } P_{\max }\right)}{P_{\max }} \leq k_{\max }
$$

where in this case $P_{\max }=\beta^{g_{\max }} P_{\min }$, thus;

$$
\frac{g_{\max }}{\beta^{g_{\max }}}+g_{\max } \leq k_{\max }
$$

which leads to;

$$
\beta \geq\left(\frac{g_{\max }}{k_{\max }-g_{\max }}\right)^{\frac{1}{\operatorname{gmax}}}
$$

Since total power should be minimized, which means minimum power ratio among groups, hence one can write;

$$
\beta_{\min }=\left(\frac{g_{\max }}{k_{\max }-g_{\max }}\right)^{\frac{1}{\max }}
$$

where $g_{\max }<k_{\max }$. Knowing that $g_{\max }$ and $k_{\max }$ are integers, and $g_{\max }$ should have maximum value; thus $g_{\max }=k_{\max }-1$. Substituting for $g_{\max }$;

$$
\beta_{\min }=\left(k_{\max }-1\right)^{\left(\frac{1}{k_{\max }-1}\right)}
$$

It is obvious that one can find a solution for power allocation problem for any FEC code by knowing its $k_{\max }$, which is the objective of this research. However, by knowing that every FEC code has its own $k_{\max }$, which may be somewhat difficult to find; hence it is preferable if one can make further simplification to equation (6).

\subsection{Simplification of equation (6)}

In order to understand (6), it is preferable to study its characteristics. Figure 6 shows the variation of the minimum power ratio $\beta_{\min }$ versus range of $k_{\max }$ integer values. One can observe that for $k_{\max }>4$ (which is the case in practical FEC codes), increasing $k_{\max }$ always results in decreasing the power ratio $\beta_{\text {min }}$ . This will in turn reduce the total power. Although larger values of $\beta_{\text {min }}$ ensure iterative receiver convergence to the target BER with fewer iterations, but it will be at the expense of unwanted increase in total power. Thus, a reasonable trade-off should be achieved. 


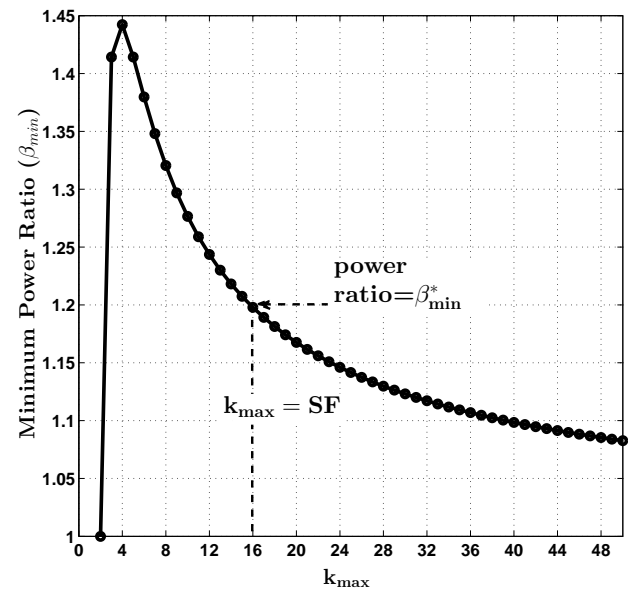

Figure 6. $\beta_{\min }$ vs. $k_{\max }$

In order to make a proper trade-off, Figure 3 should be studied carefully. By looking from the line $N=16=S F$, one can note that strong FEC codes such as FEC1 and FEC2 have $k_{\max }<S F$. Whereas weak FEC codes (such as FEC3) have $k_{\max }>S F$. Thus, it may be suitable to consider $S F$ as the average value for $k_{\max }$ for a range of FEC codes; from the strongest one to weakest.

According to the previous discussion, an acceptable trade-off can be achieved by replacing $k_{\max }$ in (6) by $S F$. This replacement leads to simplify (6) to;

$$
\beta_{\text {min }}^{*} \approx(S F-1)^{\left(\frac{1}{S F-1}\right)}
$$

Thus, in order to find the power ratio $\beta_{\min }^{*}$ we only need to know the total spreading factor ( $S F=$ $1 / R_{c} R_{p}$ ) rather than knowing $k_{\max }$ for each code. So, generally speaking, to find the minimum power ratio $\Psi_{\min }$ for certain group size $(g)$, the rule can be rewritten in the following final general form;

$$
\left.\Psi_{\text {min }}\right|_{g}=\left(\beta_{\text {min }}^{*}\right)^{g} \approx(S F-1)^{\left(\frac{g}{S F-1}\right)}
$$

It is worth mentioning that the rule in equation (8) is derived under assumption that the users who undergo the power control process should utilize the same total FEC rate.

\subsection{Proposed power allocation algorithm}

Figure 7 shows the proposed power allocation algorithm. It starts by entering total number of users, total FEC rate, and the minimum transmit power allowed for each user. Then an initial value for group size $(g)$ is assumed to be 1 . After that, the algorithm runs to find the group size that satisfies the minimum total power; then allocates the power among users accordingly.

\section{RESULTS AND DISCUSSION}

To verify the ability of our proposed method to solve the power allocation problem, the method has been applied to different FEC codes. For the sake of simplicity, FEC3 is discussed. Figure 8 shows two methods of finding the power ratio; our proposed method (green color) and one of the conventional methods for NOMA (brown color) [1,2,4,6-10,28] compared to the corresponding optimum method (blue color). Compared with the optimal results, the proposed method seems in good agreement. However, saying "in good agreement" can not be considered as a measure. So, one should put a measure to describe the degree of agreement precisely. Thus, in this paper a term coincidence $(C)$ has been used to represent this measure of agreement; more generally the percentage coincidence which can be expressed as $C=(1-\epsilon) \times 100 \%$, where;

$$
\begin{gathered}
\epsilon=\frac{\Psi_{\text {estimated }}-\Psi_{\text {optimum }}}{\Psi_{\text {optimum }}} \\
C=\left(1-\left(\frac{\Psi_{\text {estimated }}-\Psi_{\text {optimum }}}{\Psi_{\text {optimum }}}\right)\right) \times 100 \%
\end{gathered}
$$




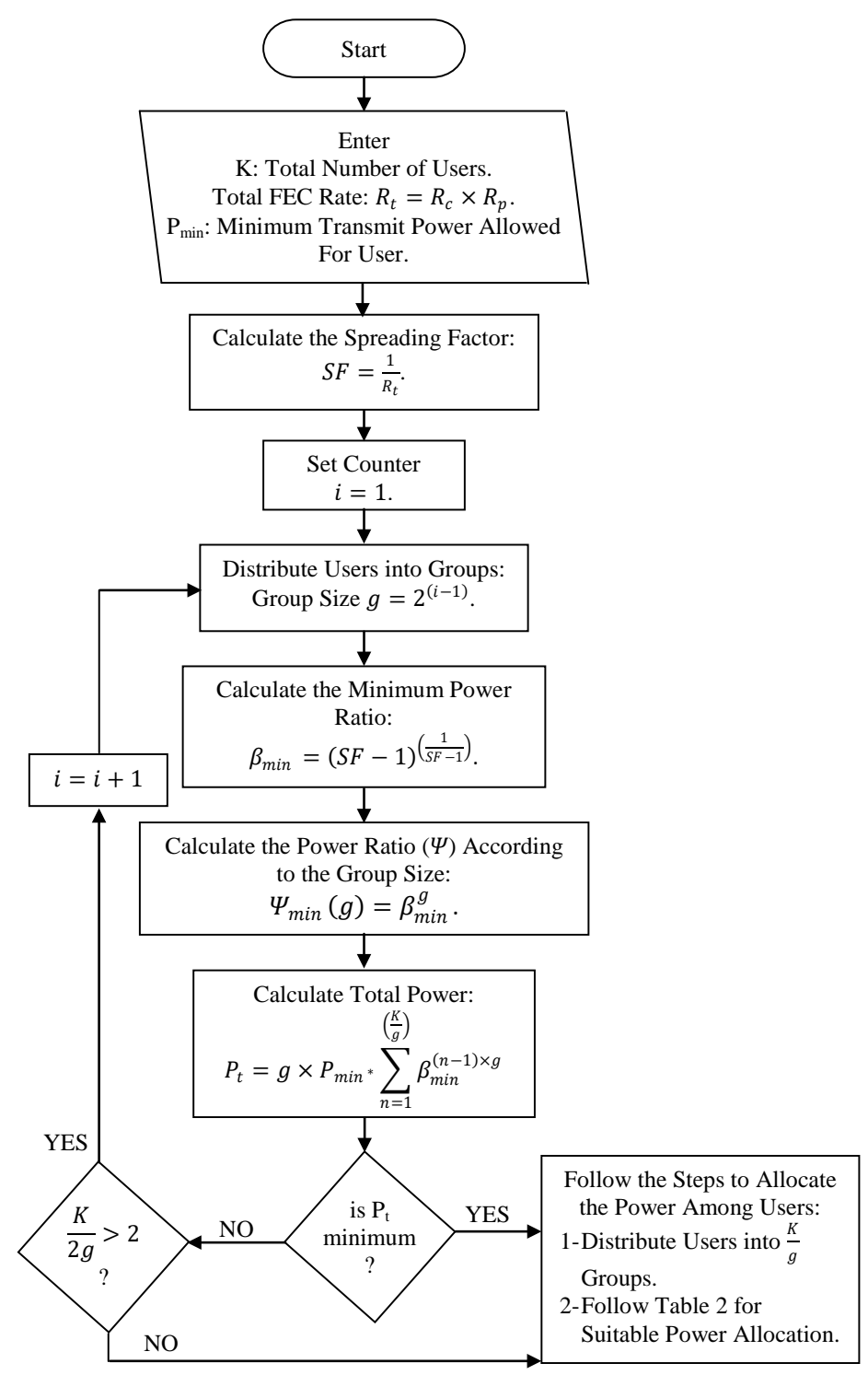

Figure 7. Proposed power allocation algorithm

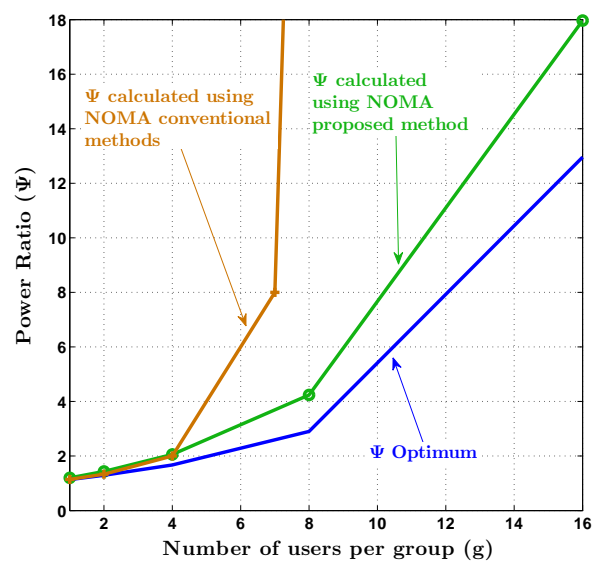

Figure 8. Comparison among different methods of finding the power ratio for FEC3 
Figure 9 illustrates the degree of coincidence of the proposed method as compared to the conventional one. It is clear that for the conventional method, the coincidence $(C)$ is excellent for $g=4$ or fewer; whereas it drops down for $g$ larger than 4. However, our proposed method shows better performance especially for $g$ larger than 4. Accordingly, one may decide to distribute users into small groups (low values of $g$ ) for the sake of maximum coincidence. However, in the power allocation problem some other parameters must be taken into account. One of these, is the total power consumed by active users $\left(P_{t}\right)$. It is known that the purpose behind the power allocation is to distribute power among users to facilitate the multi-user detection process, with minimum total power consumption. Thus, one should choose minimum $P_{t}$. Figure 10 illustrates that our proposed method can achieve a minimum total power of $25 \mathrm{~dB}$ at $g=16$, whereas the conventional method achieves $27 d B$ at $g=1$.

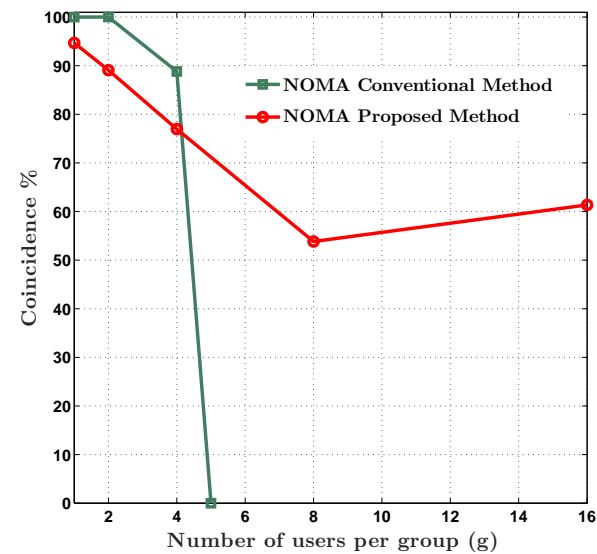

Figure 9. Percentage coincidence vs. $g$ for FEC3

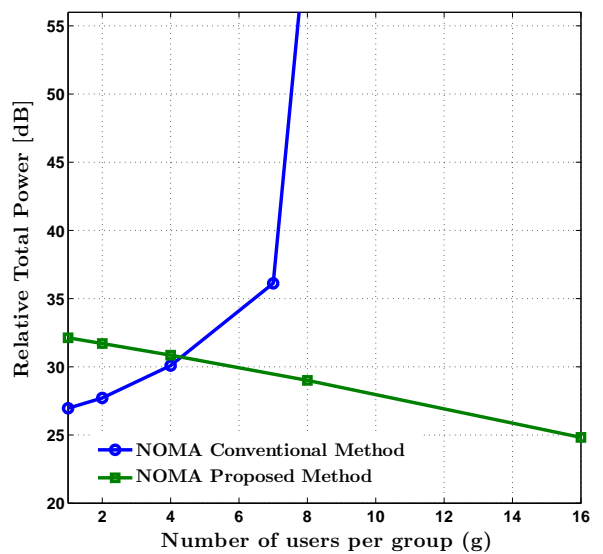

Figure 10. Relative total power vs. $g$ for FEC3

It is clear that our proposed method reduces the total power by $2 d B$ at $g=16$. According to Figure $8, g=16$ corresponds to significant increase in the power ratio (green color) as compared to the optimum one. Fortunately, this increase will facilitate the detection process by reducing the number of iterations needed to reach the target BER as shown in Figure 11.

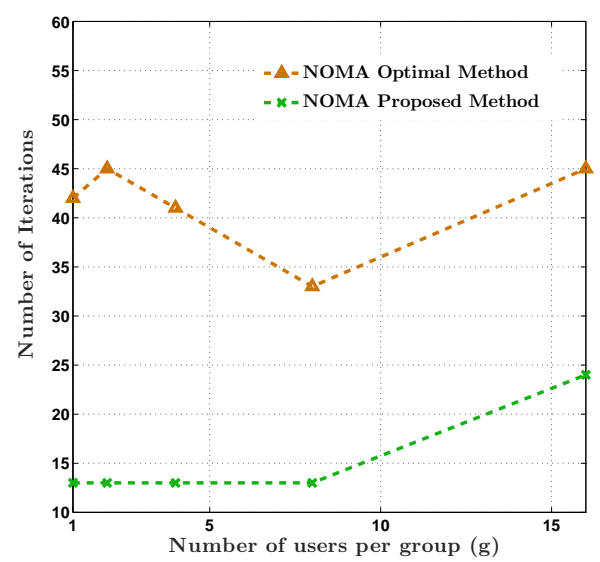

Figure 11. Receiver iterations vs. group size. A comparison between the proposed method and the simulation results for FEC3

It is important here to mention that the 'optimum method' denotes to the method that achieves the minimum total power regardless the number of receiver iterations required to reach target BER. Now, referring to Figure 11, our proposed method shows considerable reduction in the number of iterations over the possible values of $g$. This can be interpreted as follows: from the iterative receiver point of view, the increase in the power ratio $\Psi$ increases the receiver ability to discriminate among users groups. This in turn 
enhances the receiver ability to converge to the target BER $\left(10^{-6}\right)$ with fewer number of iterations. In other words, it means reducing the time for receiver to achieve its assigned task. Figure 12 illustrates the percentage reduction in the time required by the receiver to converge to the target BER which is called here 'Task Time Reduction'. It is clear that choosing $g=16$ can reduce task time by $47 \%$.

Moreover, to further verify the validity of our proposed method; we have applied it to different FEC codes. Figure 13 and Figure 14 show the relative total power graphs for FEC2 and FEC1, respectively. It is clear that our proposed method outperforms the conventional one by increasing the FEC code constraint length. In other words, the proposed method performs better as the FEC code becomming stronger.

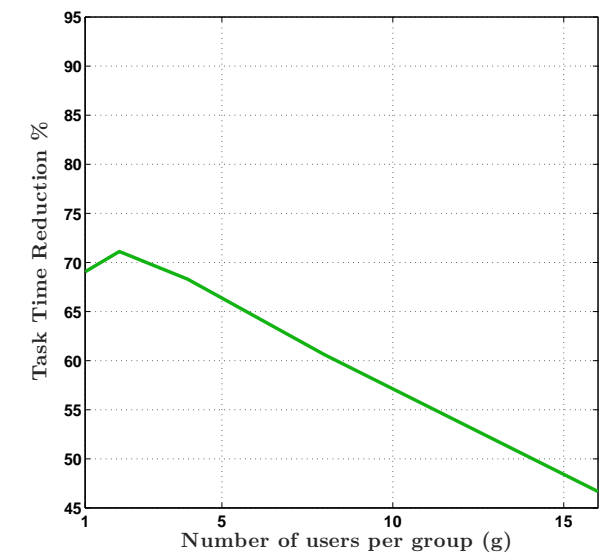

Figure 12. The percentage Task Time reduction vs. $g$ for FEC3

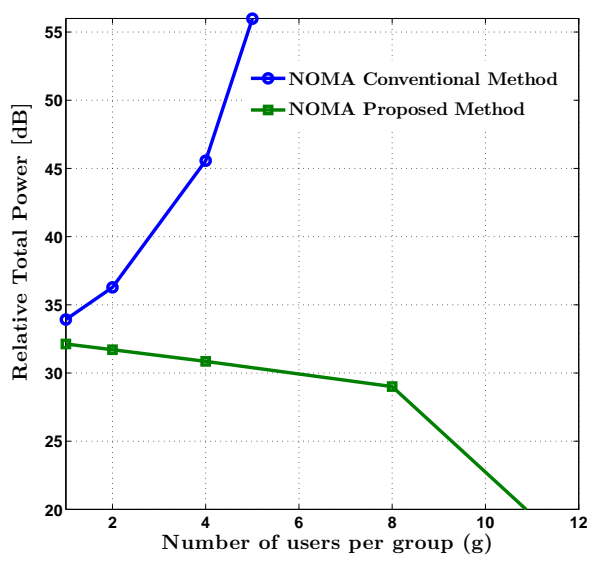

Figure 13. Relative total power vs. $g$ for FEC2

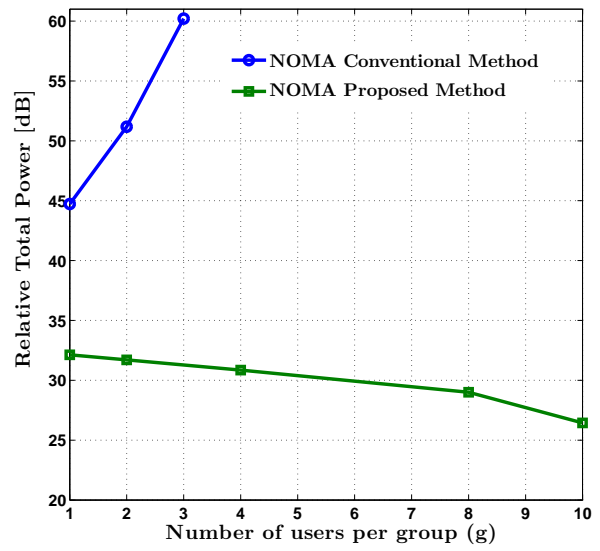

Figure 14. Relative total power vs. $g$ for FEC1

\section{CONCLUSIONS}

In this paper, a new method has been derived to simplify the solution of the power allocation problem in one of the promising NOMA systems; that is the IDMA system. Compared with the conventional methods, the proposed method is proved to be efficient and easy to apply because it needs only total system FEC rate to find a solution to the power allocation problem. Finally, the obtained numerical results show that this simple method is more feasible than its counterparts and could be applied right away.

\section{REFERENCES}

[1] J. Zhu, et al., "On optimal power allocation for downlink non-orthogonal multiple access systems," IEEE Journal on Sel. Areas in Comm., vol. 35, no. 12, pp. 2744-2757, 2017. 
[2] M. S. Ali, et al., "Downlink power allocation for CoMP-NOMA in multi-cell networks," IEEE Trans. on Comm., vol. 66, no. 9, pp. 3982-3998, 2018.

[3] Z. Ding, P. Fan, and H. V. Poor, "Impact of user pairing on 5g nonorthogonal multiple-access downlink transmissions," IEEE Trans. on Vehicular Tech., vol. 65, no. 8, pp. 6010-6023, 2016.

[4] E. R. Rezagah, T. Yoshioka, and N. Suzuki, "Power allocation for uplink non-orthogonal multiple access in 5G," 2017 International Conf. on Wireless Comm., Signal Proc. and Networking (WiSPNET), pp. 16-20, 2017.

[5] K. Senel and S. Tekinay, "Optimal power allocation in noma systems with imperfect channel estimation," 2017 IEEE Global Comm. Conf. (GLOBECOM 2017), pp. 1-7, 2017.

[6] F. Fang, J. Cheng, and Z. Ding, "Joint energy efficient subchannel and power optimization for a downlink NOMA heterogeneous network," IEEE Trans. on Vehicular Tech., vol. 68, no. 2, pp. 1351-1364, 2019.

[7] W. U. Khan, et al., "Efficient power allocation in downlink multi-cell multi-user NOMA networks," IET Comm., vol. 13, no. 4, pp. 396-402, 2019.

[8] X. Chen, et al., "Optimal power allocations for non-orthogonal multiple access over 5G full/half-duplex relaying mobile wireless networks," IEEE Trans. on Wireless Comm., vol. 18, no. 1, pp. 77-92, 2019.

[9] S. Ma, et al., "Optimal power allocation for mobile users in non-orthogonal multiple access visible light communication networks," IEEE Trans. on Comm., vol. 67, no. 3, pp. 2233-2244, 2019.

[10] L. Ping and L. Liu, "Analysis and design of IDMA systems based on snr evolution and power allocation," IEEE 60th Vehicular Tech. Conf. VTC2004-Fall, vol. 2, pp. 1068-1072, 2004.

[11] L. Ping, "Interleave division multiple-access," IEEE Trans. on Wireless Comm., vol. 5, no. 4, pp. 938-947, 2006.

[12] Z. Rosberg, "Optimal transmitter power control in interleave division multiple access (IDMA) spread spectrum uplink channels," IEEE Trans. on Wireless Comm., vol. 6, no. 1, pp. 192-201, 2007.

[13] P. Weitkemper and K. D. Kammeyer, "Power optimization of IDMA systems with different target BER constraints," 2007 IEEE 65th Vehicular Tech. Conf. VTC2007-Spring, pp. 2812-2816, 2007.

[14] M. S. K. Lau, W. Yue, and P. Wang, "On a power allocation method for IDMA systems," IEEE Trans. on Wireless Comm., vol. 7, no. 8, pp. 2905-2910, 2008.

[15] P. Wang, J. Xiao, and L. Ping, "Comparison of orthogonal and nonorthogonal approaches to future wireless cellular systems," IEEE Veh. Tech. Mag., vol. 1, no. 3, pp. 4-11, 2006.

[16] Y. Chen, "Exploiting gaussian approximation for non-orthogonal coded access," 2018 IEEE 87th Vehicular Tech. Conf. (VTC Spring), pp. 1-5, 2018.

[17] R. Keating, R. Ratasuk, and A. Ghosh, "Investigation of non-orthogonal multiple access techniques for future cellular networks," 2017 IEEE 86th Vehicular Tech. Conf. (VTC-Fall), pp. 1-5, 2017.

[18] P. Marsch, et al., "5G radio access network architecture: Design guidelines and key considerations," IEEE Comm. Mag., vol. 54, no. 11, pp. 24-36, 2016.

[19] L. Dai, et al., "Non-orthogonal multiple access for 5G: Solutions, challenges, opportunities, and future research trends," IEEE Comm. Mag., vol. 53, no. 9, pp. 74-81, 2015.

[20] I. M. Mahafeno, C. Langlais, and C. Jego, "OFDM-IDMA versus IDMA with ISI cancellation for quasistatic rayleigh fading multipath channels," 4th Int. Symp. Turbo Codes and Related Topics; 6th International ITG-Conf. on Source and Channel Coding, pp. 1-6, 2006.

[21] K. Kusume, G. Bauch, and W. Utschick, "IDMA vs.CDMA: Analysis and comparison of two multiple access schemes," IEEE Trans. on Wireless Comm., vol. 11, no. 1, pp. 78-87, 2012.

[22] P. Hammarberg, F. Rusek, and O. Edfors, "Channel estimation algorithms for OFDM-IDMA: Complexity and performance," IEEE Trans. on Wireless Comm., vol. 11, no. 5, pp. 1722-1732, 2012.

[23] C. Novak, G. Matz, and F. Hlawatsch, "IDMA for the multiuser MIMO-OFDM uplink: A factor graph framework for joint data detection and channel estimation," IEEE Trans. on Signal Proc., vol. 61, no. 16, pp. 4051-4066, 2013.

[24] K. Wu, K. Anwar, and T. Matsumoto, "BICM-ID-based IDMA using extended mapping," IEICE Trans. on Comm., vol. E97-B, no. 7, pp. 1483-1492, 2014.

[25] L. Liu, et al., "Quantize-and-forward strategy for interleave-division multiple-access relay channel," IEEE Trans. Veh. Tech., vol. 65, no. 3, pp. 1808-1814, 2016.

[26] L. Bing, et al., "Design and performance analysis of multiuser CPM with single user detection complexity," IEEE Trans. on Wireless Comm., vol. 15, no. 6, pp. 4032-4044, 2016.

[27] G. Song and J. Cheng, "Distance enumerator analysis for interleave division multi-user codes," IEEE 
Trans. on Info. Theory, vol. 62, no. 7, pp. 4039-4053, 2016.

[28] C. Shlegel, Z. Zhenning, and M. Burnashev, "Optimal power/rate allocation and code selection for iterative joint detection of coded random CDMA," IEEE Trans. on Info. Theory, vol. 52, no. 9, pp. 4286-4294,2006.

[29] P. A. Hoeher and H. Schoeneich, "Interleave-division multiple access from a multiuser theory point of view," 4th Int. Symp. on Turbo Codes and Related Topics; 6th International ITG-Conf. on Source and Channel Coding, pp. 1-5, 2006.

\section{BIOGRAPHY OF AUTHOR}

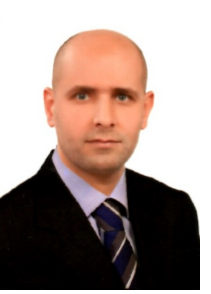

Maan Ahmed Shehathah Al-Adwany born in Mosul/Iraq in August 1974. In 1997 he received his BSc degree from College of Engineering, University of Mosul, Electrical Engineering (Electronics and Communications), also he received his MSc degree in Electronics and Communications Engineering from the same institution in year 2000. He worked as assistant lecturer at Mosul University since 2001, and then is promoted to a lecturer degree in 2007 and to assistant professor degree in 2013. He published more than 20 papers in international journals and conferences. Furthermore, he was working as consulting member for the Engineering Consulting Bureau of the University of Mosul in many engineering industrial projects. 